PRL-TH-94/7

UNIL-TP-2/94

March 1994

\title{
Effect of longitudinal electron polarization in the measurement of the $\tau$ weak dipole moment in $e^{+} e^{-}$collisions
}

\author{
B. Ananthanarayan \\ Institut de Physique Théorique, Bâtiment des Sciences Physiques, \\ Université de Lausanne, CH 1015, Lausanne, Switzerland. \\ and \\ Saurabh D. Rindani \\ Theory Group, Physical Research Laboratory \\ Navrangpura, Ahmedabad 380009, India
}

\begin{abstract}
$\underline{\text { Abstract }}$
We show that certain CP-odd momentum correlations in the production and subsequent decay of tau pairs in $e^{+} e^{-}$collisions get enhanced when the electron beam is longitudinally polarized. Analytic expressions for these correlations are obtained when $\tau^{+} \tau^{-}$have a "weak" dipole form factor (WDFF) coupling to $Z$ in the case of two-body decay modes of the $\tau$. Expressions of the variance of these correlations due to the standard model interactions are also presented. For $e^{+} e^{-}$collisions at the $Z$ peak, a sensitivity of about $10^{-17} e \mathrm{~cm}$ for the real part of the $\tau$ WDFF and $3 \times 10^{-17} e$ $\mathrm{cm}$ for the imaginary part of the $\tau$ WDFF can be reached using $\pi$ and $\rho$ channels (which are the most sensitive channels) in $\tau$ decay, with $10^{6} Z$ 's likely to be available at the SLC at Stanford with $e^{-}$polarization of $62 \%-75 \%$.
\end{abstract}




\section{Introduction}

The study of CP-odd correlations has been proposed in the past as a test of CP [1]. The observation of such correlations, for example in $e^{+} e^{-}$or $p \bar{p}$ experiments, would signal violation of CP. In particular, CP-odd observables arising due to a possible $\tau$ lepton electric or "weak" dipole moment have been analyzed in great detail [2,3]. Such dipole moments arise in extensions of the standard model (SM) with $\mathrm{CP}$ violation coming necessarily from sectors other than the standard $3 \times 3$ Cabibbo-KobayashiMaskawa matrix in order to be numerically significant.

Recent experiments at the LEP collider in CERN have also put an experimental upper limit [4] on the real part of the weak dipole form factor (WDFF) of $\tau$ at the $Z$ resonance by looking for the tensor correlation $T_{i j}=\left(\mathbf{q}_{+}-\mathbf{q}_{-}\right)_{i}\left(\mathbf{q}_{+} \times \mathbf{q}_{-}\right)_{j}$, where $\mathbf{q}_{+}\left(\mathbf{q}_{-}\right)$represents the momentum of a charged particle arising from the decay of $\tau^{+}\left(\tau^{-}\right)$produced in $Z$ decay.

Recently, the Stanford Linear Collider (SLC) has achieved a longitudinal polarization $\left(P_{e}\right)$ of $62 \%$ for the electron beam, which is likely to be increased to about $75 \%$ [5]. Longitudinal polarization of $e^{-}$of $22 \%$ has already been used for the determination of $\sin ^{2} \theta_{W}$ using left-right asymmetry [6]. Experiments with the present polarization of $62 \%$ can lead to better accuracy than obtained so far at LEP [7].

We study here the effect of longitudinal $e^{-}$polarization on CP-odd momentum correlations in $e^{+} e^{-} \rightarrow \tau^{+} \tau^{-}$with the subsequent decay of $\tau^{+}$and $\tau^{-}$. Since we have mainly the ongoing experiments at SLC in mind, we concentrate on the $e^{+} e^{-}$centreof-mass (c.m.) energy tuned to the $Z$ resonance. CP violating effects give rise to $\mathrm{CP}$-odd correlations among $\tau$ momenta and spins. In practice, tau spin is measured by looking at its decay products, and we need consider only the directly observable momentum correlations. The CP-odd momentum correlations are associated with the c.m. momenta $\mathbf{p}$ of $e^{+}, \mathbf{q}_{\overline{\mathbf{B}}}$ of $\bar{B}$ and $\mathbf{q}_{\mathbf{A}}$ of $A$, where the $\bar{B}$ and $A$ arise in the decays $\tau^{+} \rightarrow \bar{B}+\bar{\nu}_{\tau}$ and $\tau^{-} \rightarrow A+\nu_{\tau}$, where $A, B$ run over $\pi, \rho, A_{1}$, etc. In the case when $\mathrm{A}$ and $\mathrm{B}$ are different, one has to consider also the decays with $\mathrm{A}$ and $\mathrm{B}$ interchanged, so as to construct correlations which are explicitly CP-odd.

This work extends the work of an earlier paper [8], where we had obtained analytic expressions for the correlations in the single-pion decay mode of the tau. Besides including more details, the calculations of [8] are now generalized to include other two-body decay modes of the $\tau$ in general and is applied specifically to the case of $\tau \rightarrow \pi+\nu_{\tau}$ and $\tau \rightarrow \rho+\nu_{\tau}$ due to the fact that these modes possess a good resolving power of the $\tau$ polarization, parametrized in terms of the constant $\alpha$ which takes the value 1 for the $\pi$ channel (with branching fraction of about 11\%) and 0.46 for the $\rho$ channel [9] (with branching fraction of about $22 \%$ ). It may be noted that with these final states the substantive fraction of the channels that are sensitive to such correlations are accounted for; three body leptonic final states must also be included; they are characterized by a somewhat smaller $\alpha=-0.33$ (with branching fraction 
of about 35\%). Thus with the channels studied here, one more or less reaches the limits of discovery in such experiments. (It would also be possible to apply this to the decay $\tau \rightarrow A_{1}+\nu ; \alpha_{A_{1}}$ is however too small to be of any experimental relevance.) Further, we also present closed form expressions for the variance of the correlations considered due to standard model interactions. These, because of finite statistics, provide a measure of the $\mathrm{CP}$-invariant background to the determination of the $\mathrm{CP}$ odd contributions to the correlations. In case of a negative result, the limit on the $\mathrm{CP}$-violating interactions is obtained using the value of the variance and the size of the data sample.

It must be noted that correlations which are $\mathrm{CP}$ violating in the absence of initial beam polarization are not strictly $\mathrm{CP}$ odd for arbitrary $e^{+}$and $e^{-}$polarizations, since the initial state is then not necessarily $\mathrm{CP}$ even. We argue, however, that this is true to a high degree of accuracy in the case at hand.

Our main result is that certain CP-odd correlations, which are relatively small in the absence of $e^{-}$polarization, since they come with a factor $r=2 g_{V e} g_{A e} /\left(g_{V e}^{2}+g_{A e}^{2}\right)$ $(\approx 0.16)$, get enhanced in the presence of polarization, now being proportional to $\left(r-P_{e}\right) /\left(1-r P_{e}\right)\left(\approx 0.71\right.$ for $\left.P_{e}=-.62\right)[10]$. Here $g_{V e}, g_{A e}$ are the vector and axial vector couplings of $e^{-}$to $Z$. The correlations which have this property are those which have an odd number of factors of the $e^{+}$c.m. momentum $\mathbf{p}$, since this would need $\mathrm{P}$ and $\mathrm{C}$ violation at the electron vertex. Furthermore, we suggest a procedure for obtaining these correlations from the difference in the event distributions for a certain polarization $P_{e}$ and the sign-flipped polarization $-P_{e}$. With this procedure, the correlations are further enhanced, leading to increased sensitivity. The inclusion of the $\rho$ channel leads to a considerable improvement in the sensitivity that can be reached in the measurement of Im- $\tilde{d}_{\tau}$ while improving the measurement of Re- $\tilde{d}_{\tau}$ less spectacularly. It is once again worth noting that these simple vector correlations can probe both the magnitude and phase of the WDFF in the presence of large polarization despite the modest luminosity of SLC to accuracies comparable to (or even better than) those of the tensor correlations suggested in [3].

More specifically, we have considered the observables $O_{1} \equiv \frac{1}{2}\left[\hat{\mathbf{p}} \cdot\left(\mathbf{q}_{\bar{B}} \times \mathbf{q}_{A}\right)\right.$ $\left.+\hat{\mathbf{p}} \cdot\left(\mathbf{q}_{B} \times \mathbf{q}_{\bar{A}}\right)\right]$ and $O_{2} \equiv \frac{1}{2}\left[\hat{\mathbf{p}} \cdot\left(\mathbf{q}_{A}+\mathbf{q}_{\bar{B}}\right)+\hat{\mathbf{p}} \cdot\left(\mathbf{q}_{\bar{A}}+\mathbf{q}_{B}\right)\right]$ (the caret denoting a unit vector) and obtained analytic expressions for their mean values and standard deviations in the presence of longitudinal $e^{-}$polarization $P_{e}$. By the procedure outlined above, the WDFF at the $Z, \tilde{d}_{\tau}\left(m_{Z}\right)$ which can be measured at $1 \sigma$ level is about $5 \times 10^{-17} e \mathrm{~cm}\left(10^{-17} e \mathrm{~cm}\right)$ for a sample of $50,000\left(10^{6}\right)$ Z's, using these two-body $\tau$ decay channel. Moreover, $\mathrm{O}_{2}$, being CPT-odd, measures $\operatorname{Im} \tilde{d}_{\tau}$, whereas $O_{1}$ measures $\operatorname{Re} \tilde{d}_{\tau}$. Inclusion of other exclusive $\tau$ decay modes (not studied here) would improve the sensitivity further. 


\section{Notation and Formalism}

Although much of this section has already been described in our previous paper [8] we will repeat it for the sake of completeness and to make the generalization from the $\pi$ channel case more transparent.

The process we consider is

$$
e^{-}\left(p_{-}\right)+e^{+}\left(p_{+}\right) \rightarrow \tau^{-}\left(k_{-}\right)+\tau^{+}\left(k_{+}\right),
$$

with the subsequent decays

$$
\tau^{-}\left(k_{-}\right) \rightarrow A\left(q_{A}\right)+\nu_{\tau}, \tau^{+}\left(k_{+}\right) \rightarrow \bar{B}\left(q_{\bar{B}}\right)+\bar{\nu}_{\tau},
$$

together with decays corresponding to $A$ and $B$ interchanged in (2).

Under $\mathrm{CP}$, the various three-momenta transform as

$$
\mathbf{p}_{-} \leftrightarrow-\mathbf{p}_{+}, \mathbf{k}_{-} \leftrightarrow-\mathbf{k}_{+}, \mathbf{q}_{A, B} \leftrightarrow-\mathbf{q}_{\bar{A}, \bar{B}}
$$

We choose for our analysis the two CP-odd observables $O_{1} \equiv \frac{1}{2}\left[\hat{\mathbf{p}} \cdot\left(\mathbf{q}_{\bar{B}} \times \mathbf{q}_{A}\right)\right.$ $\left.+\hat{\mathbf{p}} \cdot\left(\mathbf{q}_{B} \times \mathbf{q}_{\bar{A}}\right)\right]$ and $O_{2} \equiv \frac{1}{2}\left[\hat{\mathbf{p}} \cdot\left(\mathbf{q}_{A}+\mathbf{q}_{\bar{B}}\right)+\hat{\mathbf{p}} \cdot\left(\mathbf{q}_{\bar{A}}+\mathbf{q}_{B}\right)\right]$, which have an odd number of factors of $\hat{\mathbf{p}}$, the unit vector along $\mathbf{p}_{+}$. As mentioned before, they are expected to get enhanced in the presence of $e^{-}$polarization.

Though these observables are CP odd, their observation with polarized $e^{+}$and $e^{-}$beams is not necessarily an indication of $\mathrm{CP}$ violation, unless the $e^{+}$and $e^{-}$ longitudinal polarizations are equal and opposite, so that the initial state is described by a CP-even density matrix. The case we consider here, namely, when only the $e^{-}$ is polarized, therefore needs further discussion.

In the case when only the electron beam is polarized, the CP-invariant interactions can in principle contribute to the correlations we have calculated. However, in the limit of $m_{e}=0$, the couplings of like-helicity $e^{+} e^{-}$pairs to spin-1 states like $\gamma$ and $Z$ drop out, effectively giving rise to a CP-even initial state to a very good accuracy for arbitrary $e^{+}$and $e^{-}$polarizations. The exact extent to which CP-invariant interactions would contribute to the correlations would be model dependent, and has to be calculated. However, it is clear that at the $Z$ resonance, the corrections would be suppressed at least by a factor $m_{e} / m_{Z} \approx 6 \times 10^{-6}$.

There are however corrections at order $\alpha$ from tree-level helicity-flip collinear photon emission which are not suppressed by electron mass $[11,12]$. We have not considered any order- $\alpha$ corrections in this work. However, if there is a CP-invariant background at order $\alpha$ to the correlations we calculate, this could completely wipe out the small CP-violating effects we are concerned about. Fortunately, the helicity-flip cross section being non-resonant is suppressed by a factor of about $10^{-4}$ in the region of the resonance [12]. It is therefore expected that the corresponding correlations will be small enough not to affect our sensitivity estimates, and can be neglected. (In 
fact, for the case of our observable $O_{1}$ which is T odd, we need not worry about this, since CP invariant contributions necessarily need to have an absorptive part, which is not present in the tree-level order- $\alpha$ correction).

There may be higher-order non-resonant CP-odd helicity combinations (without collinear photon emission) which contribute to $\left\langle O_{1,2}\right\rangle$ at one loop. Since these do not interfere with the leading $Z$ contributions coming from CP-even helicity combinations, they will be suppressed by a factor $\alpha^{2} \Gamma_{Z}^{2} / m_{Z}^{2} \approx 5 \times 10^{-8}$.

Of $O_{1}$ and $O_{2}, O_{1}$ is even under the combined CPT transformation, and $O_{2}$ is CPT-odd. A CPT-odd observable can only have a non-zero value in the presence of an absorptive part of the amplitude. It is therefore expected that $\left\langle\mathrm{O}_{2}\right\rangle$ will be proportional to the imaginary part of the weak dipole form factor $\operatorname{Im} \tilde{d}_{\tau}$, since finalstate interaction, which could give rise to an absorptive part, is negligible in the weak $\tau$ decays. Since $\left\langle O_{1}\right\rangle$ and mean values of other CPT-even quantities will be proportional to $\operatorname{Re} \tilde{d}_{\tau}$, phase information on $\tilde{d}_{\tau}$ can only be obtained if $\left\langle O_{2}\right\rangle$ (or some other CPT-odd quantity) is also measured.

We assume SM couplings for all particles except $\tau$, for which an additional WDFF interaction is assumed, viz.,

$$
\mathcal{L}_{W D F F}=-\frac{i}{2} \tilde{d}_{\tau} \bar{\tau} \sigma^{\mu \nu} \gamma_{5} \tau\left(\partial_{\mu} Z_{\nu}-\partial_{\nu} Z_{\mu}\right)
$$

where $\tilde{d}_{\tau} \equiv \tilde{d}_{\tau}\left(s=m_{Z}^{2}\right)$. Using (4), we now proceed to calculate $\left\langle O_{1}\right\rangle$ and $\left\langle O_{2}\right\rangle$ in the presence of longitudial polarization $P_{e}$ for $e^{-}$.

We can anticipate the effect of $P_{e}$ in general for the process (1). We can write the matrix element squared for the process in the leading order in perturbation theory, neglecting the electron mass, as

$$
|M|^{2}=\sum_{i, j} L_{\mu \nu}^{i j}(e) L^{i j \mu \nu *}(\tau) \frac{1}{s-M_{i}^{2}} \frac{1}{s-M_{j}^{2}},
$$

where the summation is over the gauge bosons $(\gamma, Z, \ldots)$ exchanged in the $s$ channel, and $L_{\mu \nu}^{i j}(e, \tau)$ represent the tensors arising at the $e$ and $\tau$ vertices:

$$
L_{\mu \nu}^{i j}=V_{\mu}^{i} V_{\nu}^{j *}
$$

For the electron vertex, with only the SM vector and axial vector couplings,

$$
V_{\mu}^{i}(e)=g_{i} \bar{v}\left(p_{+}, s_{+}\right) \gamma_{\mu}\left(g_{V e}^{i}-\gamma_{5} g_{A e}^{i}\right) u\left(p_{-}, s_{-}\right),
$$

$g_{i}$ being the appropriate coupling constant $\left(g_{\gamma}=e, g_{Z}=g /\left(2 \cos \theta_{W}\right)\right)$, and $g_{V e}^{i}$ and $g_{A e}^{i}$ are given by

$$
\begin{gathered}
g_{V e}^{\gamma}=-1, g_{A e}^{\gamma}=0 \\
g_{V e}^{Z}=-\frac{1}{2}+2 \sin ^{2} \theta_{W}, g_{A e}^{Z}=-\frac{1}{2} .
\end{gathered}
$$


It is easy to check, by putting in helicity projection operators, that

$$
\begin{aligned}
& L_{\mu \nu}^{i j}(e)=g_{i} g_{j} \\
& \quad \times\left\{\left[\left(1-P_{e} P_{\bar{e}}\right)\left(g_{V e}^{i} g_{V e}^{j}+g_{A e}^{i} g_{A e}^{j}\right)-\left(P_{e}-P_{\bar{e}}\right)\left(g_{V e}^{i} g_{A e}^{j}+g_{A e}^{i} g_{V e}^{j}\right)\right] \operatorname{Tr}\left(\not p_{-} \gamma_{\mu} \not p_{+} \gamma_{\nu}\right)\right. \\
& \left.\quad+\left[\left(P_{e}-P_{\bar{e}}\right)\left(g_{V e}^{i} g_{V e}^{j}+g_{A e}^{i} g_{A e}^{j}\right)-\left(1-P_{e} P_{\bar{e}}\right)\left(g_{V e}^{i} g_{A e}^{j}+g_{A e}^{i} g_{V e}^{j}\right)\right] \operatorname{Tr}\left(\gamma_{5} \not p_{-} \gamma_{\mu} p_{+} \gamma_{\nu}\right)\right\}
\end{aligned}
$$

in the limit of vanishing electron mass, where $P_{e}\left(P_{\bar{e}}\right)$ is the degree of the $e^{-}\left(e^{+}\right)$ longitudinal polarization. Note that the combinations $P_{e}-P_{\bar{e}}$ and $1-P_{e} P_{\bar{e}}$ occurring in (10) are indeed CP-even, showing that the initial state is effectively CP even for arbitrary $P_{e}, P_{\bar{e}}$.

Eq.(10) gives a simple way of incorporating the effect of the longitudinal polarization. In particular, at the $Z$ peak, where photon effects can be neglected, to go from the unpolarized to the polarized one, one has to make the replacement (henceforth, we drop the superscript $Z$, as we shall only deal with $Z$ couplings):

$$
\begin{gathered}
g_{V e}^{2}+g_{A e}^{2} \rightarrow g_{V e}^{2}+g_{A e}^{2}-P_{e} 2 g_{V e} g_{A e}, \\
2 g_{V e} g_{A e} \rightarrow 2 g_{V e} g_{A e}-P_{e}\left(g_{V e}^{2}+g_{A e}^{2}\right) .
\end{gathered}
$$

We have set $P_{\bar{e}}=0$, as is the case at SLC, for example. It is then clear from (9), using $\sin ^{2} \theta_{W} \approx 0.23$, that quantities which are suppressed in the absence of polarization because of the small numerical value of $2 g_{V e} g_{A e}$ will get considerably enhanced in the presence of polarization.

To calculate correlations of $O_{1}$ and $O_{2}$, we need the differential cross section for (1) followed by (2) at the $Z$ peak, arising from SM $Z$ couplings of $e$ and $\tau$, together with a weak-dipole coupling of $\tau$ arising from eq.(4). (We neglect electromagnetic effects completely). The calculation may be conveniently done, following ref.[3] (see also ref.[13]), in steps, by first determining the production matrix $\chi$ for $\tau^{+} \tau^{-}$in spin space, and then taking its trace with the decay matrices $\mathcal{D}^{ \pm}$for $\tau^{ \pm}$decays into single charged particle in addition to the invisible neutrino.

$$
\frac{1}{\sigma} \frac{d \sigma}{d \Omega_{k} d \Omega_{-}^{*} d \Omega_{+}^{*} d E_{-}^{*} d E_{+}^{*}}=\frac{k}{8 \pi m_{Z}^{2} \Gamma\left(Z \rightarrow \tau^{+} \tau^{-}\right)} \frac{1}{(4 \pi)^{3}} \chi^{\beta \beta^{\prime}, \alpha \alpha^{\prime}} \mathcal{D}_{\alpha^{\prime} \alpha}^{-} \mathcal{D}_{\beta^{\prime} \beta}^{+},
$$

where $d \Omega_{k}$ is the solid angle element for $\mathbf{k}_{+}$in the overall c.m. frame, $k=\left|\mathbf{k}_{+}\right|$, and $d \Omega_{ \pm}^{*}$ are the solid angle elements for $\mathbf{q}_{\bar{B}, A}^{*}$, the $\bar{B}$ and $A$ momenta in the $\tau^{ \pm}$rest frame. The $\mathcal{D}$ matrices are given by

$$
\begin{aligned}
& \mathcal{D}^{+}=\delta\left(E_{B}^{*}-E_{0 B}\right)\left[1-\alpha_{B} \sigma_{+} \cdot \hat{\mathbf{q}}_{B}^{*}\right] \\
& \mathcal{D}^{-}=\delta\left(E_{A}^{*}-E_{0 A}\right)\left[1+\alpha_{A} \sigma_{-} \cdot \hat{\mathbf{q}}_{A}^{*}\right],
\end{aligned}
$$

where $\sigma_{ \pm}$are the Pauli matrices corresponding to the $\tau^{ \pm}$spin, $E_{ \pm}^{*}$ are the charged particle energies in the $\tau^{ \pm}$rest frame, and

$$
E_{0 A, B}=\frac{1}{2} m_{\tau}\left(1+p_{A, B}\right) ; p_{A, B}=m_{A, B}^{2} / m_{\tau}^{2} .
$$


The expressions for $\chi$ arising from SM as well as WDFF coupling of $\tau$ are rather long, and we refer the reader to ref.[3] for these expressions in the absence of polarization. It is straightforward to incorporate polarization using (11).

\section{Results}

Using eqns. (13)-(15) above, as well as the expression for the $\tau^{+} \tau^{-}$production matrix $\chi$ from [3], we can obtain expressions for $\left\langle O_{1}\right\rangle$ and $\left\langle O_{2}\right\rangle$ by writing $O_{1}$ and $O_{2}$ in terms of the $\tau$ rest frame variables and carrying out integrals over them analytically. The expressions for the correlations $\left\langle O_{1}\right\rangle$ and $\left\langle O_{2}\right\rangle$ obtained are, neglecting $\tilde{d}_{\tau}^{2}$,

$$
\begin{gathered}
\left\langle O_{1}\right\rangle=-\frac{m_{Z}^{2}}{18 e} m_{\tau} \operatorname{Re} \tilde{d}_{\tau} c_{W} s_{W}\left(1-x^{2}\right)\left(\frac{r-P_{e}}{1-r P_{e}}\right) \\
\frac{g_{A \tau} \alpha_{A} \alpha_{B}\left(1-p_{A}\right)\left(1-p_{B}\right)-\frac{3}{2} g_{V \tau}\left[\alpha_{A}\left(1-p_{A}\right)\left(1+p_{B}\right)+\alpha_{B}\left(1-p_{B}\right)\left(1+p_{A}\right)\right]}{g_{V \tau}^{2}\left(1+\frac{1}{2} x^{2}\right)+g_{A \tau}^{2}\left(1-x^{2}\right)}
\end{gathered}
$$

and

$$
\begin{gathered}
\left\langle O_{2}\right\rangle=\frac{2 m_{Z}}{3 e} m_{\tau} \operatorname{Im} \tilde{d}_{\tau} c_{W} s_{W}\left(\frac{r-P_{e}}{1-r P_{e}}\right) \\
\frac{g_{A \tau}\left(1-x^{2}\right)^{2} \frac{1}{2}\left(\alpha_{A}\left(1-p_{A}\right)+\alpha_{B}\left(1-p_{B}\right)\right)}{g_{V \tau}^{2}\left(1+\frac{1}{2} x^{2}\right)+g_{A \tau}^{2}\left(1-x^{2}\right)}
\end{gathered}
$$

where $c_{W}=\cos \theta_{W}, s_{W}=\sin \theta_{W}, x=2 m_{\tau} / m_{Z}$, and $r=2 g_{V e} g_{A e} /\left(g_{V e}^{2}+g_{A e}^{2}\right)$.

We have also obtained analytic expressions for the variance $\left\langle O^{2}\right\rangle-\langle O\rangle^{2} \approx\left\langle O^{2}\right\rangle$ in each case, arising from the CP-invariant SM part of the interaction:

$$
\begin{gathered}
\left\langle O_{1}^{2}\right\rangle=\frac{3}{64} \frac{1}{\left(g_{V_{\tau}}^{2}\left(1+\frac{1}{2} x^{2}\right)+g_{A_{\tau}}^{2}\left(1-x^{2}\right)\right)} m_{Z}^{2} m_{\tau}^{2} \\
\left(\frac{1}{270}\left(1-p_{A}\right)^{2}\left(1-p_{B}\right)^{2}\left[g_{V_{\tau}}^{2}\left(12+16 x^{2}+2 x^{4}\right)+g_{A_{\tau}}^{2}\left(12-4 x^{2}-8 x^{4}\right)\right]\right. \\
+\frac{\left(1-x^{2}\right)}{540}\left(\left[\left(1+p_{A}\right)^{2}\left(1-p_{B}\right)^{2}+\left(1+p_{B}\right)^{2}\left(1-p_{A}\right)^{2}\right]\right. \\
\left.\left[12 g_{V_{\tau}}^{2}\left(3+4 x^{2}\right)\right)+36 g_{A_{\tau}}^{2}\left(1-x^{2}\right)\right] \\
\left.+16 \alpha_{A} \alpha_{B}\left(1-p_{B}^{2}\right)\left(1-p_{A}^{2}\right)\left(1-x^{2}\right)\left[g_{V_{\tau}}^{2}-g_{A_{\tau}}^{2}\right]\right) \\
+\frac{4}{45}\left(1-p_{A}\right)\left(1-p_{B}\right) g_{V_{\tau}} g_{A_{\tau}}\left(1-x^{2}\right)\left(1-\frac{x^{2}}{6}\right) \\
\left.\left[\alpha_{A}\left(1+p_{A}\right)\left(1-p_{B}\right)+\alpha_{B}\left(1+p_{B}\right)\left(1-p_{A}\right)\right]\right) \\
\left\langle O_{2}^{2}\right\rangle=\frac{1}{12} \frac{1}{\left(g_{V_{\tau}}^{2}\left(1+\frac{1}{2} x^{2}\right)+g_{A_{\tau}}^{2}\left(1-x^{2}\right)\right)} m_{Z}^{2} \\
{\left[\left(\frac{1}{10}\left[\left(1-p_{A}\right)^{2}+\left(1-p_{B}\right)^{2}\right]\left[g_{V_{\tau}}^{2}\left(1+\frac{7}{4} x^{2}+x^{4}\right)+g_{A_{\tau}}^{2}\left(1+\frac{1}{2} x^{2}-\frac{3}{2} x^{4}\right)\right]\right.\right.}
\end{gathered}
$$




$$
\begin{gathered}
\left.+\frac{1}{15} \alpha_{A} \alpha_{B}\left(1-p_{A}\right)\left(1-p_{B}\right)\left[g_{V_{\tau}}^{2}\left(-1-\frac{7}{4} x^{2}-x^{4}\right)+g_{A_{\tau}}^{2}\left(-1+2 x^{2}-x^{4}\right)\right]\right) \\
+\frac{9}{4}\left(\frac{2}{15}\left(1-x^{2}\right)\left(p_{A}-p_{B}\right)^{2}\left[g_{V_{\tau}}^{2}\left(1+\frac{x^{2}}{4}\right)+g_{A_{\tau}}^{2}\left(1-x^{2}\right)\right]\right. \\
\left.\left.-\frac{2}{45} g_{V_{\tau}} g_{A_{\tau}}\left(1-x^{2}\right)^{2}\left(4+x^{2}\right)\left(p_{A}-p_{B}\right)\left[\alpha_{A}\left(1-p_{A}\right)-\alpha_{B}\left(1-p_{B}\right)\right]\right)\right]
\end{gathered}
$$

The results for the significant two-body decay channels are presented in the tables. In Tables 1 and 2 we have presented, as in ref.[3], the values of $c_{A B}$ for $O_{1}$ and $O_{2}$ respectively, defined as the correlation for a value of $\operatorname{Re} \tilde{d}_{\tau}$ or $\operatorname{Im} \tilde{d}_{\tau}$ (as the case may be) equal to $e / m_{Z}$, for various values of $P_{e}$. We have also presented the value of $\sqrt{\left\langle O^{2}\right\rangle}$ and $\delta \tilde{d}_{\tau}$, which represents the 1 s.d. upper limit unit on $\tilde{d}_{\tau}$ which can be placed with a certain sample of events, for 50,000 Z's currently seen at SLC with $62 \%$ polarization, and for $10^{6} Z$ 's, eventually hoped to be achieved. This 1 s.d. limit is the value of $\tilde{d}_{\tau}$ which gives a mean value of $O_{i}$ equal to the s.d. $\sqrt{\left\langle O_{i}^{2}\right\rangle / N_{A B}}$ in each case:

$$
c_{A B} \delta \tilde{d}_{\tau}=\frac{e}{m_{Z}} \frac{1}{\sqrt{N_{A B}}} \sqrt{\left\langle O_{i}^{2}\right\rangle} .
$$

Here $N_{A B}$ is the number of events in the channel $A \bar{B}$ (or $\bar{A} B$ ), and is given by

$$
N_{A B}=N_{Z} B\left(Z \rightarrow \tau^{+} \tau^{-}\right) B\left(\tau^{-} \rightarrow A \nu_{\tau}\right) B\left(\tau^{+} \rightarrow \bar{B} \bar{\nu}_{\tau}\right)
$$

As can be seen from Tables 1 and $2,\left\langle O_{1}\right\rangle$ and $\left\langle O_{2}\right\rangle$ can probe respectively $\operatorname{Re} \tilde{d}_{\tau}$ and $\operatorname{Im} \tilde{d}_{\tau}$ down to about $1.4 \times 10^{-16} e \mathrm{~cm}$ and $4.1 \times 10^{-16} e \mathrm{~cm}$ with the current data from SLC, where we have added the sensitivities by inverse quadrature to obtain these sensitivities. These limits can be improved by a factor of 5 in future runs of SLC.

These limits can be improved by looking at correlations of the same observables, but in a sample obtained by counting the difference between the number of events for a certain polarization, and for the corresponding sign-flipped polarization. If the partial cross section for the process for an $e^{-}$polarization $P_{e}$ is given by

$$
d \sigma\left(P_{e}\right)=A+P_{e} \cdot B
$$

then one calculates average values over the distribution given by the difference

$$
d \sigma\left(P_{e}\right)-d \sigma\left(-P_{e}\right)=2 P_{e} B
$$

The correlations are considerably larger, whereas the variances are unchanged. The event sample is however smaller (by a factor of about $.16 P_{e}$, which is the left-right asymmetry times degree of polarization), leading to a larger statistical error. The sensitivity is nevertheless improved, as can be seen from Table 3. There we present, for the two variables $O_{1}$ and $O_{2}, c_{A B}$ (defined as before) for the polarization asymmetrized sample described above, and the corresponding quantities $\sqrt{\left\langle O_{i}^{2}\right\rangle}$ and the 1 s.d. limits on $\operatorname{Re} \tilde{d}_{\tau}$ and $\operatorname{Im} \tilde{d}_{\tau}$, respectively, both called $\delta \tilde{d}_{\tau}$ in this table for convenience. We 
give the limits for two luminosities, but only for one polarization, $P_{e}=.62$. Only the last column changes with $P_{e}$, and the improvement in going to $P_{e}=.75$ is marginal.

As seen from Table 3, the current data can yield upper limits of about $5.0 \times 10^{-17} e$ $\mathrm{cm}$ for $\operatorname{Re} \tilde{d}_{\tau}$ and $1.5 \times 10^{-16} e \mathrm{~cm}$ for $\operatorname{Im} \tilde{d}_{\tau}$, where we have again combined errors from different channels by adding inverse squares. An integrated luminosity corresponding to $10^{6}$ Z's at SLC can improve the limits to about $1.2 \times 10^{-17} \mathrm{e} \mathrm{cm}$ and $3.3 \times 10^{-17} \mathrm{e} \mathrm{cm}$, respectively. These should be compared with the $95 \%$ C.L. ALEPH limit of Re $\tilde{d}_{\tau}$ $<3.7 \times 10^{-17} e \mathrm{~cm}$ obtained by looking for tensor correlations from a sample of about 650,000 Z's at LEP, and including several decay channels of $\tau$ [4]. The disarming simplicity of the correlations considered is reflected in the fact that analytic expressions presented above yield results comparable to those from more involved correlations involving expensive numerical methods and the necessity of including several decays. The simple two-body channels considered here more or less set the scale of the limits of discover since they have larger sensitivity than the leptonic and three-body channels. Further the expressions given here would serve as a normalization tool for a Monte Carlo package should it be constructed.

The real advantage of polarization can be seen in the sensitivity for the measure-

ment of $\operatorname{Im} \tilde{d}_{\tau}$. The limit obtainable at LEP under ideal experimental conditions from the $2 \pi$ channel using the tensor correlation $\left\langle\hat{\mathbf{p}} \cdot\left(\hat{\mathbf{q}}_{+}+\hat{\mathbf{q}}_{-}\right) \hat{\mathbf{p}} \cdot\left(\hat{\mathbf{q}}_{+}-\hat{\mathbf{q}}_{-}\right)\right\rangle$is $10^{-16} \mathrm{e} \mathrm{cm}$ with $10^{7} Z$ 's [3], whereas the limit we obtain here in the presence of polarization is as low as $5 \times 10^{-17} \mathrm{e} \mathrm{cm}$ with only $10^{6} Z$ 's for the same channel.

\section{Conclusions}

To conclude, our results show that the CP-odd correlations $\left\langle O_{1}\right\rangle$ and $\left\langle O_{2}\right\rangle$ are considerably enhanced due to longitudinal $e^{-}$polarization. By considering values of these correlations on changing the sign of the polarization, a much greater sensitivity is obtained. Considering both these correlations together can give phase information on the WDFF, which cannot be obtained using any single correlation. While the sensitivity obtainable at SLC for the real part of the WDFF is comparable to that obtained at LEP, the sensitivity for the imaginary part is better than that obtainable at LEP with an ordinary of magnitude lower luminosity at SLC.

We have neglected errors in measurement of $P_{e}$, whose effect on the results would be small compared to that of statistical errors. We have also ignored radiative corrections, which may alter our numerical estimates somewhat, and must be taken into account in a more complete analysis.

It is possible that longitudinal polarization may be available at tau-charm factories or other future linear colliders planned to operate at higher energies. The advantages of polarization presented here can be studied by extending the analysis to the relevant energies, including the effect of photon exchange and the $\tau$ electric dipole coupling. 
The study could also be extended to dipole moments of the top-quark or $W^{ \pm}$which could be pair produced at the high energy accelerators. The more complete study presented here will serve as a useful cross-check on Monte-Carlos used in the evaluation of the correlations and their variances. Further, in the event of a very massive sequential lepton found say at the JLC which is likely to have several two-body decay modes, the expressions given here can be easily modified to evaluate the CP-violating form factors of such a lepton, albeit with the interference from an electric-dipole form factor since the Z-cross section would no longer dominate.

It would be worthwhile to calculate order- $\alpha$ corrections to the results obtained above. However, this would be model dependent, and requires considerable care and effort.

It would also be interesting to consider event asymmetries corresponding to $O_{1}$ and $\mathrm{O}_{2}$ (rather than their mean values), and also other CP-odd correlations with an odd number of $\hat{\mathbf{p}}$ 's, like $\left\langle\left(\mathbf{q}_{+}^{2}-\mathbf{q}_{-}^{2}\right) \hat{\mathbf{p}} \cdot\left(\mathbf{q}_{+}-\mathbf{q}_{-}\right)\right\rangle$, which may have a different sensitivity.

\section{Acknowledgements}

B. A. thanks Prof. G. Wanders for a conversation. Support from the Swiss National Science Foundation during the course of this work for B. A. is gratefully acknowledged. S.D.R. thanks D. Indumathi, Anjan Joshipura, Marek Nowakowski and B.R. Sitaram for discussions. He also thanks Prof. L.M. Sehgal for discussions and drawing his attention to refs. [11] and [12]. 


\section{References}

[1] J.F. Donoghue and G. Valencia, Phys. Rev. Lett. 58, 451 (1987); M. Nowakowski and A. Pilaftsis, Mod. Phys. Lett. A 4, 829 (1989), Z. Phys. C 42, 449 (1989); W. Bernreuther, U. Löw, J.P. Ma and O. Nachtmann, Z. Phys. C 43, 117 (1989); W. Bernreuther and O. Nachtmann, Phys. Lett. B 268, 424 (1991); M.B. Gavela et al., Phys. Rev. D 39, 1870 (1989).

[2] F. Hoogeveen and L. Stodolsky, Phys. Lett. B 212, 505 (1988); S. Goozovat and C.A. Nelson, Phys. Lett. B 267, 128 (1991); G. Couture, Phys. Lett. B 272, 404 (1991); W. Bernreuther and O. Nachtmann, Phys. Rev. Lett. 63, 2787 (1989).

[3] W. Bernreuther, G.W. Botz, O. Nachtmann and P. Overmann, Z. Phys. C 52, 567 (1991); W. Bernreuther, O. Nachtmann and P. Overmann, Phys. Rev. D 48, 78 (1993).

[4] OPAL Collaboration, P.D. Acton et al., Phys. Lett. B 281, 405 (1992); ALEPH Collaboration, D. Buskulic et al., Phys. Lett. B 297, 459 (1992).

[5] M. Swartz, private communication.

[6] SLD Collaboration, K. Abe et al., Phys. Rev. Lett. 70, 2515 (1993).

[7] M.J. Fero, SLAC report SLAC-PUB-6027 (1992), to be published.

[8] B. Ananthanarayan and S. D. Rindani, Physical Research Laboratory preprint, PRL-TH-93/17

[9] K. Hagiwara, A. D. Martin and D. Zeppenfeld, Phys. Lett. B 235, 198 (1990);

See also W. Bernreuther, O. Nachtmann and P. Overmann in Ref. [3].

[10] A similar enhancement for the observation of $\mathrm{CP}$ violation in $\mathrm{B}$ decays was pointed out by W.B. Atwood, I. Dunietz and P. Grosse-Wiesmann, Phys. Lett. B 216, 227 (1989).

[11] T.D. Lee and M. Nauenberg, Phys. Rev. B 133, 1549 (1964); F. Berends et al., Nucl. Phys. B239, 382,395 (1984); H.F. Contopanangos and M.B. Einhorn, Nucl. Phys. B377, 20 (1992).

[12] B. Falk and L.M. Sehgal, Aachen report no. PITHA 93/29 (Rev.), to appear in Phys. Lett. B.

[13] Y.S. Tsai, Phys. Rev. D4, 2821 (1971); 13, 771 (1976) (E); S. Kawasaki, T. Shirafuji and Y.S. Tsai, Prog. Theo. Phys. 49, 1656 (1973). 


\section{Tables}

1. Correlations and 1 s.d. limits on Re $\tilde{d}_{\tau}$ for the observable $O_{1}$ corresponding to the final states (a) $\pi \pi(\mathrm{b}) \pi \rho$ and (c) $\rho \rho$.

2. Correlations and 1 s.d. limits on $\operatorname{Im} \tilde{d}_{\tau}$ for the observable $O_{2}$ corresponding to the final states (a) $\pi \pi(\mathrm{b}) \pi \rho$ and (c) $\rho \rho$.

3. Results for the polarization asymmetrized sample of events for $P_{e}=0.62$. Here $\delta \tilde{d}_{\tau}$ refers to its real part in case of $O_{1}$ and to its imaginary part for $O_{2}$. 


\begin{tabular}{||c|c|c|c|c||}
\hline$P_{e}$ & $c_{\pi \pi}$ & $\sqrt{\left\langle O_{1}^{2}\right\rangle}$ & \multicolumn{2}{|c||}{$\delta \operatorname{Re} \tilde{d}_{\tau}(e \mathrm{~cm})$ for } \\
\cline { 4 - 6 } & $\left(\mathrm{GeV}^{2}\right)$ & $\left(\mathrm{GeV}^{2}\right)$ & $5 \times 10^{4} Z$ 's & $10^{6} Z$ 's \\
\hline 0 & 0.898 & 12.861 & $6.6 \times 10^{-16}$ & $1.5 \times 10^{-16}$ \\
+0.62 & -2.890 & 12.861 & $2.0 \times 10^{-16}$ & $4.6 \times 10^{-17}$ \\
-0.62 & 4.007 & 12.861 & $1.5 \times 10^{-16}$ & $3.3 \times 10^{-17}$ \\
+0.75 & -3.792 & 12.861 & $1.6 \times 10^{-16}$ & $3.5 \times 10^{-17}$ \\
-0.75 & 4.589 & 12.861 & $1.3 \times 10^{-16}$ & $2.9 \times 10^{-17}$ \\
\hline
\end{tabular}

Table 1(a)

\begin{tabular}{||c|c|c|c|c||}
\hline$P_{e}$ & $c_{\pi \rho}$ & $\sqrt{\left\langle O_{1}^{2}\right\rangle}$ & \multicolumn{2}{|c||}{$\delta \operatorname{Re} \tilde{d}_{\tau}(e \mathrm{~cm})$ for } \\
\cline { 4 - 5 } & $\left(\mathrm{GeV}^{2}\right)$ & $\left(\mathrm{GeV}^{2}\right)$ & $5 \times 10^{4} Z$ 's & $10^{6} Z$ 's \\
\hline 0 & 0.223 & 13.433 & $2.0 \times 10^{-15}$ & $4.5 \times 10^{-16}$ \\
+0.62 & -0.716 & 13.433 & $6.2 \times 10^{-16}$ & $1.4 \times 10^{-16}$ \\
-0.62 & 0.993 & 13.433 & $4.5 \times 10^{-16}$ & $1.0 \times 10^{-16}$ \\
+0.75 & -0.940 & 13.433 & $4.8 \times 10^{-16}$ & $1.1 \times 10^{-16}$ \\
-0.75 & 1.137 & 13.433 & $3.9 \times 10^{-16}$ & $8.8 \times 10^{-17}$ \\
\hline
\end{tabular}

Table 1(b)

\begin{tabular}{||c|c|c|c|c||}
\hline$P_{e}$ & $c_{\rho \rho}$ & $\sqrt{\left\langle O_{1}^{2}\right\rangle}$ & \multicolumn{2}{|c||}{$\delta \operatorname{Re} \tilde{d}_{\tau}(e \mathrm{~cm})$ for } \\
\cline { 4 - 6 } & $\left(\mathrm{GeV}^{2}\right)$ & $\left(\mathrm{GeV}^{2}\right)$ & $5 \times 10^{4} Z$ 's & $10^{6} Z$ 's \\
\hline 0 & 0.040 & 12.874 & $7.7 \times 10^{-15}$ & $1.7 \times 10^{-15}$ \\
+0.62 & -0.130 & 12.874 & $2.4 \times 10^{-15}$ & $5.4 \times 10^{-16}$ \\
-0.62 & 0.180 & 12.874 & $1.7 \times 10^{-15}$ & $3.9 \times 10^{-16}$ \\
+0.75 & -0.170 & 12.874 & $1.8 \times 10^{-15}$ & $4.1 \times 10^{-16}$ \\
-0.75 & 0.206 & 12.874 & $1.5 \times 10^{-15}$ & $3.4 \times 10^{-16}$ \\
\hline
\end{tabular}

Table 1(c) 


\begin{tabular}{||c|c|c|c|c||}
\hline$P_{e}$ & $c_{\pi \pi}$ & $\sqrt{\left\langle O_{2}^{2}\right\rangle}$ & \multicolumn{2}{|c||}{$\delta \operatorname{Im} \tilde{d}_{\tau}(e \mathrm{~cm})$ for } \\
\cline { 4 - 5 } & $(\mathrm{GeV})$ & $(\mathrm{GeV})$ & $5 \times 10^{4} Z$ 's & $10^{6} Z$ 's \\
\hline 0 & -0.157 & 9.572 & $2.8 \times 10^{-15}$ & $6.2 \times 10^{-16}$ \\
+0.62 & 0.505 & 9.572 & $8.7 \times 10^{-16}$ & $1.9 \times 10^{-16}$ \\
-0.62 & -0.700 & 9.572 & $6.3 \times 10^{-16}$ & $1.4 \times 10^{-16}$ \\
+0.75 & 0.662 & 9.572 & $6.6 \times 10^{-16}$ & $1.5 \times 10^{-16}$ \\
-0.75 & -0.802 & 9.572 & $5.5 \times 10^{-16}$ & $1.2 \times 10^{-16}$ \\
\hline
\end{tabular}

Table 2(a)

\begin{tabular}{||c|c|c|c|c||}
\hline$P_{e}$ & $c_{\pi \rho}$ & $\sqrt{\left\langle O_{2}^{2}\right\rangle}$ & \multicolumn{2}{|c||}{$\delta \operatorname{Im} \tilde{d}_{\tau}(e \mathrm{~cm})$ for } \\
\cline { 4 - 6 } & $(\mathrm{GeV})$ & $(\mathrm{GeV})$ & $5 \times 10^{4} Z$ 's & $10^{6} Z$ 's \\
\hline 0 & -0.108 & 10.324 & $3.2 \times 10^{-15}$ & $7.1 \times 10^{-16}$ \\
+0.62 & 0.347 & 10.324 & $9.9 \times 10^{-16}$ & $2.2 \times 10^{-16}$ \\
-0.62 & -0.482 & 10.324 & $7.1 \times 10^{-16}$ & $1.6 \times 10^{-16}$ \\
+0.75 & 0.456 & 10.324 & $7.5 \times 10^{-16}$ & $1.7 \times 10^{-16}$ \\
-0.75 & -0.552 & 10.324 & $6.2 \times 10^{-16}$ & $1.4 \times 10^{-16}$ \\
\hline
\end{tabular}

Table 2(b)

\begin{tabular}{||c|c|c|c|c||}
\hline$P_{e}$ & $c_{\rho \rho}$ & $\sqrt{\left\langle O_{2}^{2}\right\rangle}$ & \multicolumn{2}{|c||}{$\delta \operatorname{Im} \tilde{d}_{\tau}(e \mathrm{~cm})$ for } \\
\cline { 4 - 5 } & $(\mathrm{GeV})$ & $(\mathrm{GeV})$ & $5 \times 10^{4} Z$ 's & $10^{6} Z$ 's \\
\hline 0 & -0.059 & 9.246 & $3.8 \times 10^{-15}$ & $8.4 \times 10^{-16}$ \\
+0.62 & 0.190 & 9.246 & $1.2 \times 10^{-15}$ & $2.6 \times 10^{-16}$ \\
-0.62 & -0.264 & 9.246 & $8.5 \times 10^{-16}$ & $1.9 \times 10^{-16}$ \\
+0.75 & 0.249 & 9.246 & $8.9 \times 10^{-16}$ & $2.0 \times 10^{-16}$ \\
-0.75 & -0.302 & 9.246 & $7.4 \times 10^{-16}$ & $1.7 \times 10^{-16}$ \\
\hline
\end{tabular}

Table 2(c) 


\begin{tabular}{||c|c|c|c|c||}
\hline & $c_{\pi \pi}$ & $\sqrt{\left\langle O_{i}^{2}\right\rangle}$ & \multicolumn{2}{|c||}{$\delta \tilde{d}_{\tau}(e \mathrm{~cm})$ for } \\
\cline { 4 - 5 } & & & $5 \times 10^{4} Z$ 's & $10^{6} Z$ 's \\
\hline$O_{1}$ & $35.545 \mathrm{GeV}^{2}$ & $12.861 \mathrm{GeV}^{2}$ & $5.3 \times 10^{-17}$ & $1.2 \times 10^{-17}$ \\
$O_{2}$ & $-6.208 \mathrm{GeV}$ & $9.572 \mathrm{GeV}$ & $2.2 \times 10^{-16}$ & $5.0 \times 10^{-17}$ \\
\hline
\end{tabular}

Table 3(a)

\begin{tabular}{||c|c|c|c|c||}
\hline & $c_{\pi \rho}$ & $\sqrt{\left\langle O_{i}^{2}\right\rangle}$ & \multicolumn{2}{|c||}{$\delta \tilde{d}_{\tau}(e \mathrm{~cm})$ for } \\
\cline { 4 - 5 } & & & $5 \times 10^{4} Z$ 's & $10^{6} Z$ 's \\
\hline$O_{1}$ & $8.810 \mathrm{GeV}^{2}$ & $13.433 \mathrm{GeV}^{2}$ & $1.6 \times 10^{-16}$ & $3.6 \times 10^{-17}$ \\
$O_{2}$ & $-4.273 \mathrm{GeV}$ & $10.324 \mathrm{GeV}$ & $2.6 \times 10^{-16}$ & $5.7 \times 10^{-17}$ \\
\hline
\end{tabular}

Table 3(b)

\begin{tabular}{||c|c|c|c|c||}
\hline & $c_{\rho \rho}$ & $\sqrt{\left\langle O_{i}^{2}\right\rangle}$ & \multicolumn{2}{|c||}{$\delta \tilde{d}_{\tau}(e \mathrm{~cm})$ for } \\
\cline { 4 - 5 } & & & $5 \times 10^{4} Z^{\prime} \mathrm{s}$ & $10^{6} Z^{\prime} \mathrm{s}$ \\
\hline$O_{1}$ & $1.594 \mathrm{GeV}^{2}$ & $12.874 \mathrm{GeV}^{2}$ & $6.2 \times 10^{-16}$ & $1.4 \times 10^{-16}$ \\
$O_{2}$ & $-2.338 \mathrm{GeV}$ & $9.246 \mathrm{GeV}$ & $3.0 \times 10^{-16}$ & $6.8 \times 10^{-17}$ \\
\hline
\end{tabular}

Table 3(c) 